\title{
Impact of a multidisciplinary approach to ultrasound- guided thyroid fine-needle aspiration biopsy at Tygerberg Hospital, Cape Town, South Africa: A retrospective audit
}

\author{
W Conradie, ${ }^{1}$ MB ChB, MMed (Surg) (D); A du Plessis, ${ }^{2}$ MB ChB, MMed (Rad); J Edge, ${ }^{1}$ MBBS, MMed (Surg) ; K Baatjes, ${ }^{1}$ MB ChB, PhD; \\ A Ruiters, ${ }^{3} \mathrm{MB}$ ChB; R Razack, ${ }^{4} \mathrm{MB}$ ChB, MMed (Anat Path) \\ ${ }^{1}$ Division of General Surgery, Department of Surgery, Faculty of Medicine and Health Sciences, Tygerberg Hospital and Stellenbosch University, \\ Cape Town, South Africa \\ ${ }^{2}$ Division of Radiodiagnosis, Department of Medical Imaging and Clinical Oncology, Faculty of Medicine and Health Sciences, Tygerberg Hospital \\ and Stellenbosch University, Cape Town, South Africa \\ ${ }^{3}$ Faculty of Medicine and Health Sciences, Tygerberg Hospital and Stellenbosch University, Cape Town, South Africa \\ ${ }^{4}$ Division of Anatomical Pathology, National Health Laboratory Service, Faculty of Medicine and Health Sciences, Tygerberg Hospital and \\ Stellenbosch University, Cape Town, South Africa
}

Corresponding author: W Conradie (ilnaconradie@sun.ac.za)

\begin{abstract}
Background. Thyroid nodules are common and mostly benign. Inadequate sampling generally occurs in 13 - $17 \%$ of thyroid fine-needle aspiration biopsies (FNABs), but the proportion was found to be as high as $45 \%$ on evaluating 100 ultrasound (US)-guided FNABs in a previous unpublished audit at Tygerberg Hospital, Cape Town, South Africa (SA).

Objectives. Primary aim: To determine the diagnostic yield of US-guided thyroid biopsy after implementing changes to existing practices, involving the creation of a specialised clinic and applying protocols for referral and FNAB. Secondary aim: To compare the results with other centres in SA.

Methods. A retrospective audit of 178 thyroid biopsies was conducted. All US-guided thyroid biopsies performed in the specialised clinic between January 2017 and July 2018 were included. Data were analysed using descriptive statistics.

Results. The 178 nodules were biopsied in 159 patients. The mean age was 53.7 years, with a gender ratio of 9.6:1 (female/male). A reduction in non-diagnostic biopsies was noted compared with the historical cohort (45\% v. 32.6\%). Sixty-one nodules (34.3\%) had previously been biopsied with inadequate cytology results. When repeat biopsies were excluded, only $16.2 \%(n=19)$ were classified as insufficient.

Conclusions. These findings illustrate the importance of the multidisciplinary approach and standardisation of the US-guided biopsy procedure and the value of consistency and quality control in a health system. While nodular thyroid disease is common and FNAB is relatively simple, early referral to a central specialised unit to minimise the incidence of inadequate FNAB should be considered.

S Afr Med J 2022;112(1):49-52. https://doi.org/10.7196/SAMJ.2022.v112i1.15906
\end{abstract}

Thyroid nodules are common. ${ }^{[1]}$ The widespread use of sensitive imaging techniques for head, neck and chest investigations, coupled with the increasing use of high-resolution ultrasound (US), has increased the frequency of thyroid nodules being discovered. ${ }^{[2,3]}$ The prevalence ranges from $2 \%$ to $76 \%$ depending on the population screened and the detection method, the latter including palpation, imaging and autopsy data. ${ }^{[2,3]}$

All thyroid nodules carry a risk of malignancy (RoM), ranging from $5 \%$ to $15 \% .^{[1,3,4]}$ This risk is evaluated by three parameters: clinical, US and cytological assessment. Ideally, US imaging should be combined with fine-needle aspiration biopsy (FNAB). FNAB is widely accepted as the most accurate method to evaluate the RoM in thyroid nodules. However, a limitation of this technique is nondiagnostic results, which are reported in up to $29 \%$ of US-guided FNABs. $^{[3,5]}$ The resultant RoM in non-diagnostic aspirates, albeit low, is $5-10 \%{ }^{[6]}$

At Tygerberg Hospital (TBH), Cape Town, South Africa (SA), a retrospective audit of US-guided FNABs estimated the rate of non-diagnostic cytology reports to be $\sim 45 \%{ }^{\left[{ }^{[7]}\right.}$ Factors affecting the adequacy of FNAB include nodule size, vascularity, consistency, the experience of the operator, and the cytopathologist's threshold of adequacy. ${ }^{[5,7]}$ Most factors are inherent to the thyroid nodule itself, precluding improvement. However, the technique and experience of the aspirator can be addressed. This includes supervised training and rapid on-site evaluation (ROSE) of the cytological aspirate for specimen adequacy. ROSE has been shown to improve specimen yield by $83-92 \%$ and reduce the rate of non-diagnostic specimens by an average of $44 \%$. The most significant impact is noted in centres with a high rate of non-diagnostic reports. ${ }^{[8-11]}$

The Bethesda System for Reporting Thyroid Cytopathology (TBSRTC) has resolved adequacy threshold issues for pathologists. ${ }^{[6,12]}$ It was implemented at the TBH cytology laboratory and has been used since 2016. Bongiovanni's meta-analysis validated the system as accurate and reliable. ${ }^{[12]}$ The system provides a diagnostic framework of six categories with RoM for each category, assisting clinicians in further management of thyroid lesions.

Before 2017, the practice of US-guided thyroid aspiration was not standardised at TBH. Thyroid FNABs were performed by radiologists, surgeons, physicians or endocrinologists. Aspirators varied in training and experience. To create a more cohesive practice, a formal weekly thyroid clinic was established. The clinic was managed with a multidisciplinary team approach and included a dedicated surgeon, a radiologist, and a pathologist providing a ROSE service. 


\section{Objectives}

The interventional strategy aimed to improve the rate of diagnostic cytology reports and, in turn, assist surgeons in triaging patients more efficiently for surgery. In this study, we determined the impact of this intervention on the diagnostic yield of US-guided thyroid FNABs at TBH.

\section{Methods \\ Design and setting}

This retrospective audit was conducted at a multidisciplinary US-guided thyroid FNAB clinic, introduced at TBH in January 2017. As TBH functions as both a secondary and tertiary hospital, the thyroid clinic receives referrals from peripheral primary healthcare clinics, secondary hospitals, and various departments within the hospital. Protocols for indications for the biopsy, reporting of US and FNAB technique were standardised. Cytotechnologists were available for all biopsies to assist in rapid staining of FNAB slides to allow for ROSE. Guidelines from the American Thyroid Association (ATA) were followed. ${ }^{[1]}$

Thyroid cytology was reported according to the six categories described by TBSRTC: (I) non-diagnostic or unsatisfactory; (II) benign; (III) atypia of undetermined significance or follicular lesion of undetermined significance; (IV) follicular neoplasm or suspicious for a follicular neoplasm; (V) suspicious for malignancy; and (VI) malignant. ${ }^{[6]}$

\section{Participants}

All patients with radiologically or clinically suspicious nodules requiring US-guided thyroid FNAB between 1 January 2017 and 30 June 2018 were included in the study.

\section{Outcome measures}

Data collection included age, sex, number of nodules on US, size and location of the nodule, cytology result, number of previous FNABs, and final histological diagnosis if surgery was performed. Information was collected from clinical records, the National Health Laboratory Service, and the diagnostic imaging division at TBH, which uses the picture archiving and communication system.

\section{Statistical analysis}

SPSS software (SPSS Statistics 25, 2017; IBM, USA) was used for data collection and analysis.

Data were analysed using descriptive statistics. Where applicable, either Student's $t$-test or the Mann-Whitney $U$-test were used to compare the number of non-diagnostic FNAs between two groups. Results were compared with a historical cohort. ${ }^{[13]}$

\section{Ethical considerations}

The study was approved by the Undergraduate Research Ethics Committee of Stellenbosch University (ref. no. U18/03/008) and performed in accordance with the 2013 Declaration of Helsinki.

\section{Results}

One hundred and fifty-nine patients with 178 nodules were investigated by FNAB within the study period. The mean (standard deviation) age of the cohort was 53.7 (14.2) years (range 14 - 87). The gender distribution of the sample was $90.6 \%$ female and $9.4 \%$, male.

Ninety-two of the nodules (51.7\%) were in the left lobe and $80(44.9 \%)$ in the right, and 6 nodules $(3.4 \%)$ were located in the isthmus.

For assessment, the nodules were divided into two groups. Group A ( $n=61)$ had had a previous inadequate biopsy before referral. In group B $(n=117)$, the first biopsy was performed at the TBH clinic. In group A, 35 nodules (57.4\%) had one prior FNA biopsy, 12 (19.7\%) had two, $12(19.7 \%)$ had three, and $2(3.3 \%)$ had four. In group A, 39 biopsies (63.9\%) were subsequently non-diagnostic compared with only $19(16.2 \%)$ in group B. Overall, the incidence of non-diagnostic thyroid biopsies in the TBH thyroid clinic decreased from $45 \%$ to $32.6 \%(p=0.04)$. The distribution of other TBSRTC categories is shown in Table 1. Of the 178 nodules that were assessed, 57 (32.0\%) were removed surgically. The corresponding histological diagnoses are shown in Table 2.

Of resected nodules diagnosed on cytology as TBSRTC category I, most ( $n=22 ; 91.7 \%$ ) were histologically classified as benign nodular thyroid disease. One case proved to be a follicular adenoma, and $1 \mathrm{a}$ follicular variant papillary thyroid carcinoma (PTC). The RoM in this category was therefore $4.2 \%$. The RoM for the remaining categories is shown in Table 3 .

Histopathological results diagnosed 11 nodules (19.3\%) as malignant and $46(80.7 \%)$ as benign. Eight of the malignancies (72.7\%) were PTCs, of which 2 were follicular variants and one a tall cell variant. A single case of each of the following subtypes of thyroid cancer were diagnosed: follicular, medullary and poorly differentiated.

Of the 46 benign cases, $38(82.6 \%)$ were nodular disease of the thyroid. Four of the cases $(8.7 \%)$ were follicular adenomas, $1(2.2 \%)$ was a Hürthle cell adenoma, 2 (4.3\%) showed Hashimoto's thyroiditis, and $1(2.2 \%)$ was a non-invasive follicular thyroid neoplasm with papillary-like nuclear features.

Five of the benign specimens had an incidental finding of micropapillary thyroid carcinoma, and 1 incidental parathyroid adenoma was found intraoperatively and removed.

\begin{tabular}{|c|c|c|c|c|}
\hline TBSRTC category & $\begin{array}{l}\text { Total }(N=178), \\
n(\%)\end{array}$ & $\begin{array}{l}\text { Group A } \\
\text { Previous BI } \\
(N=61), n(\%)\end{array}$ & $\begin{array}{l}\text { Group B } \\
\text { First FNAB } \\
(N=117), n(\%)\end{array}$ & $\begin{array}{l}\text { Bongiovanni } \\
\text { meta-analysis }^{[12]} \\
(N=25445), n(\%)\end{array}$ \\
\hline I. Non-diagnostic or unsatisfactory & $58(32.6)$ & $39(63.9)$ & $19(16.2)$ & $3271(12.9)$ \\
\hline II. Benign & $97(54.5)$ & $12(19.7)$ & $85(72.6)$ & $15104(59.3)$ \\
\hline III. AUS/FLUS & $11(6.2)$ & $4(6.6)$ & $7(6.0)$ & $2441(9.6)$ \\
\hline IV. Follicular neoplasm or suspicious for a follicular neoplasm & $4(2.2)$ & $3(4.9)$ & $1(0.9)$ & $2571(10.1)$ \\
\hline V. Suspicious for malignancy & $2(1.1)$ & $1(1.6)$ & $1(0.9)$ & $680(2.7)$ \\
\hline VI. Malignant & $6(3.4)$ & $2(3.3)$ & $4(3.4)$ & $1378(5.4)$ \\
\hline
\end{tabular}

TBH = Tygerberg Hospital; FNAB = fine-needle aspiration biopsy; TBSRTC = The Bethesda System for Reporting Thyroid Cytopathology; BI = TBSRTC category I; AUS/FLUS = atypia of undetermined significance/follicular lesion of undetermined significance. 
Table 2. Distribution of the cytological-histological correlation of the nodules

\begin{tabular}{|c|c|c|c|c|c|c|c|c|c|}
\hline \multirow[b]{3}{*}{ TBSRTC category } & \multicolumn{8}{|c|}{ Histological follow-up } & \multirow[b]{3}{*}{ Total $(N=57)$} \\
\hline & \multicolumn{4}{|c|}{ Benign $(N=46)$} & \multicolumn{4}{|c|}{ Malignant $(N=11)$} & \\
\hline & HT & NTD & FA/HCA & NIFTP & FC & PTC & MC & PDC & \\
\hline I & 0 & 22 & 1 & 0 & 0 & $1 \mathrm{FV}$ & 0 & 0 & 24 \\
\hline II & 0 & 10 & 1 & 0 & 1 & 1 & 0 & 0 & 13 \\
\hline III & 1 & 5 & 0 & 1 & 0 & 2 & 0 & 0 & 9 \\
\hline IV & 0 & 1 & 2 (1 HCA) & 0 & 0 & $1 \mathrm{FV}$ & 0 & 0 & 5 \\
\hline $\mathrm{V}$ & 1 & 0 & 1 & 0 & 0 & 0 & 0 & 0 & 2 \\
\hline VI & 0 & 0 & 0 & 0 & 0 & 3 & 1 & 1 & 5 \\
\hline
\end{tabular}

\section{Table 3. Comparison between TBH and TBSRTC implied RoM}

\begin{tabular}{llll}
\hline \multirow{2}{*}{ TBSRTC category } & \multicolumn{2}{c}{ RoM (\%) } \\
\cline { 2 - 4 } I & TBH & TBSRTC 2009* & TBSRTC 2018 $^{\dagger}$ \\
II & 4.2 & n/a & $5-10$ \\
III & 7.6 & $0-3$ & $0-3$ \\
IV & 22.0 & $5-15$ & $10-30$ \\
V & 20.0 & $15-30$ & $25-40$ \\
VI & 0 & $60-75$ & $50-75$ \\
TBH $=$ Tygerberg Hospital; TBSRTC $=$ The Bethesda System for Reporting Thyroid Cytopathology; RoM $=$ risk of malignancy; n/a = not applicable. &
\end{tabular}

\section{Discussion}

Findings of this study show a statistically significant improvement of non-diagnostic thyroid aspirates after introducing a multidisciplinary US-guided thyroid biopsy clinic. Overall, the incidence of nondiagnostic thyroid biopsies decreased from $45 \%$ to $32.6 \%(p=0.04)$. However, the impact is probably greater, as these numbers include 61 cases $(34.3 \%)$ that were referred after a previous failed FNAB (group A in Table 1). The probability of obtaining a diagnostic aspirate following an initial non-diagnostic one decreases with every sequential attempt. ${ }^{[5]}$ However, a cytological diagnosis was made in 22 cases $(36.0 \%)$ in group A.

Once the protocol for FNABs had been implemented, most patients had their first FNA performed in the clinic. These 117 nodules form group B. The inadequacy rate of $16.2 \%$ was much lower in this group, and is comparable with international standards.

The RoM in this study was compared with the 2 nd edition of TBSRTC (Table 3). ${ }^{[6]}$ In our study, despite having small numbers, the RoM was comparable to that published in TBSRTC. RoM fell short by $5 \%$ in category IV, but the 2 cases reported as $\mathrm{V}$ were both benign. In the 2nd edition, categories III and IV have a similar RoM. Our RoM for category III is higher than for IV, supporting the proposal to condense these two categories into one tier. ${ }^{[6]}$ The inaccurate RoM in category $\mathrm{V}$ (when the expected RoM is 50 $75 \%$ ) can be explained by diagnostic interpretive error. Conversely, the two cytology cases reported as suspicious for malignancy were diagnosed histologically to be a case of lymphocytic thyroiditis and a case of follicular adenoma. Hashimoto's thyroiditis has overlapping cytological features with PTC, and in this case, an aspirate devoid of lymphocytes is a known and unavoidable pitfall in cytopathology practice. ${ }^{[14,15]}$ The false-positive cytology for the follicular adenoma can be explained by poor fixation, which can sometimes lead to misinterpretation of nuclear features. Overall, of the 57 (32.0\%) nodules diagnosed histologically, 46 (80.7\%) were found to be benign nodular disease.

The retrospective nature of the audit at a single specialist centre and the small study sample size, as well as selection bias, contribute to the limitations of the study. Patients are referred to the clinic for surgical evaluation, but it is also a referral centre for diagnostic uncertainty based on US or cytological findings. This audit does, however, provide a stepping stone for further research. In a recent review aimed at improving clinical decision-making in thyroid FNAB, Poller et al ${ }^{[8]}$ advocate local institution-driven data collection.

As much as TBSRTC provides an RoM per diagnostic category, wide inter-institutional variation exists, although little has been published from SA.

The authors of an audit of all patients undergoing thyroidectomy in Limpopo Province, SA, between 2003 and 2008 noted that they were unable to rely on cytology. ${ }^{[4]}$ A study from Groote Schuur Hospital, Cape Town, SA, reviewed all cases of thyroid malignancy seen between 2004 and 2010. ${ }^{[11]}$ The authors noted a sensitivity of $44 \%$ for cytology and suggested that the low rate was due to various factors including the inexperience of the operator, the lack of routine use of US, sampling error, and the lack of a standardised reporting system.

Recommendations for further studies include expanding the current findings by identifying clinical predictors for a non-diagnostic cytology result. Additionally, future studies could include riskstratifying surgical procedures to reduce overtreatment when thyroid nodules are found to be non-diagnostic. This could guide selection of managing patients with active surveillance principles outlined by the ATA guidelines rather than maintaining the current low threshold for diagnostic thyroid lobectomy.

Lessons learnt from this experience are that continued monitoring and surveillance of adequacy rates, cytological-histological correlation and regular multidisciplinary and interdisciplinary discussions are 
essential in providing an optimal service. Dedicated personnel and specialists concentrating on the field of concern also strengthen proficiency in the discipline.

\section{Conclusions}

We have demonstrated that implementing an initiative to improve clinical decision-making has its merits. These findings illustrate the importance of the multidisciplinary approach and standardisation of the US-guided biopsy procedure, and the value of consistency and quality control. While nodular thyroid disease is common and US-guided FNAB is relatively straightforward to do, early referral to a central specialised unit results in reduced rates of inadequacy.

\section{Declaration. None.}

Acknowledgements. The authors thank Kalien Weidemann for data capturing.

Author contributions. WC conceptionalised the study idea. WC and AR collected the data. RR, KB and JE validated the data. WC drafted the first version of the manuscript with contributions from RR and JE. All authors critically reviewed the manuscript and approved the final version.

Funding. None.

Conflicts of interest. None.

\footnotetext{
1. Haugen BR, Alexander EK, Bible KC, et al. 2015 American Thyroid Association Management Guidelines for Adult Patients with Thyroid Nodules and Differentiated Thyroid Cancer: The American Guidelines for Adult Patients with Thyroid Nodules and Differentiated Thyroid Cancer: The American
Thyroid Association Guidelines Task Force on Thyroid Nodules and Differentiated Thyroid CancerThyroid Association Guidelines Task Force on Thyroid Nodules
}

2. Dean DS, Gharib H. Epidemiology of thyroid nodules. Best Pract Res Clin Endocrinol Metab 2008;22(6):901-911. https://doi.org/10.1016/j.beem.2008.09.019

3. Gill AS, Amdur R. Joshi AS. Importance of FNA technique for decreasing non-diagnostic rates in . Gill AS, Amdur R, Joshi AS. Importance of FNA technique for decreasing non-diagnostic rates in
thyroid nodules. Head Neck Pathol 2018;12(2):160-165. https://doi.org/10.1007/s12105-017-0844-8 4. Bhuiyan MMZU, Machowski A. Nodular thyroid disease and thyroid malignancy: Experience at Polokwane Mankweng Hospital Complex, Limpopo Province, South Africa. S Afr Med J 2015;105(7):570-572. https://doi.org/10.7196/SAMJnew.7885

5. Jack GA, Sternberg SB, Aronson MD et al. Non-diagnostic fine-needle aspiration biopsy of thyroid nodules: Outcomes and determinants. Thyroid 2020;30(7):992-998. https://doi.org/10.1089/ thy. 2019.0140

6. Baloch Z, LiVolsi VA. The Bethesda System for Reporting Thyroid Cytology (TBSRTC): From lookbacks to look-ahead. Diagn Cytopathol 2020;48(10):862-866. https://doi.org/10.1002/dc.24385

7. De Koster EJ, Kist JW, Valk GD, Vriens MR, Borel Rinkes IHM, de Keizer B. Thyroid ultrasoundguided fine-needle aspiration: The positive influence of on-site adequay asesme guided fine-needle aspiration. The positive influence of on-site adequacy assessment and pecta Cytol 2016;60(1):39-45. https://doi.

org $/ 10.1159 / 000444917$
8. Poller DN, Johnson SJ, Bongiovanni M. Measures to reduce diagnostic error and improve clinical decision making in thyroid FNA aspiration cytology: A proposed framework. Cancer Cytopathol 2020;128(12):917-927. https://doi.org/10.1002/cncy.22309

9. Witt BL, Schmidt RL. Rapid on-site evaluation improves the adequacy of fine-needle aspiration for thyroid lesions: A systematic review and meta-analysis. Thyroid 2013;23(4):428-435. https://doi. org/10.1089/thy.2012.0211

10. Nicolaou MA, Jacobs K, Bhana S, Naidu K, Nicolaou V. A retrospective study correlating sonographic features of thyroid nodules with fine-needle aspiration cytology in a South African setting. S Afr J Radiol 2019;23(1):1-6. https://doi.org/10.4102/sajr.v23i1.1749

11. Cairncross L, Panieri E. Pre-operative diagnosis of thyroid cancer: Clinical, radiological and pathological correlation. S Afr J Surg 2013;51(2):46-49. https://doi.org/10.7196/sajs.1261

12. Bongiovanni M, Spitale A, Faquin WC, Mazzucchelli L, Baloch ZW. The Bethesda System for Rongiovanni M, Spitale A, Faquin WC, Mazzucchelli L, Baloch ZW. The Bethesda System for
Reporting Thyroid Cytopathology: A meta-analysis. Acta Cytol 2012;56(4):333-339. https://doi, Reporting Thyroid Cytopathology: A meta-analysis. Acta Cytol 2012;56(4):333-339. https://doi.
org/10.1159/000339959

13. Bezuidenhout $\mathrm{L}$. An audit of ultrasound-guided fine needle aspiration biopsies of thyroid nodules at an academic hospital. BTech thesis. Cape Town: Cape Peninsula University of Technology, 2016. 14. Zhu Y, Song Y, Xu G, Fan Z, Ren W. Causes of misdiagnoses by thyroid fine-needle aspiration cytology (FNAC): Our experience and a systematic review. Diagn Pathol 2020;15(1):1-8. https://doi. org/10.1186/s13000-019-0924-Z

15. Malheiros DC, Canberk S, Poller DN. Schmitt F. Thyroid FNAC: Causes of false-positive results. Cytopathology 2018;29(5):407-417. https://doi.org/10.1111/cyt.12575

Accepted 1 September 2021. 